\title{
Editorial
}

Psychotherapy and Psychosomatics

\section{Computer-Aided Cognitive Behaviour Therapy}

\author{
L. Baer ${ }^{a} \quad$ J. Greist ${ }^{b} \quad$ I.M. Marks ${ }^{c}$ \\ ${ }^{a}$ Department of Psychiatry, Harvard Medical School, Boston, Mass., and b University of Wisconsin, \\ Healthcare Technology Systems, Madison, Wisc., USA; ' Institute of Psychiatry, King's College London, \\ London, UK
}

Worldwide the demand for cognitive behaviour therapy (CBT) exceeds the availability of therapists. This gap and advances in information technology have catalyzed a rich ferment of research into computer-aided CBT (CCBT). Certain CCBT systems can save much therapist time by allowing most treatment tasks to be delegated to patient-computer interactions. In contrast, therapist time is not saved either by conducting CBT via email, telephone or videoconference exchanges between patient and therapist in real time, or via most virtual reality systems. CCBT also provides guidance personalized to each patient yet perfectly standardized given the same series of patient responses.

Fully 175 CCBT studies including 87 randomized controlled trials (RCTs) have just been reviewed in a Maudsley Monograph [1], and in this journal Emmelkamp [2] discussed technological innovations in psychotherapy including CCBT. The 97 CCBT systems reviewed in the Monograph concerned a wide spectrum of mental health problems. A growing number of CCBT systems are becoming available on the Internet. Users of CCBT on the Internet are more likely to complete it if they are first screened, are then given access by a password, and are then supported briefly by phone, email or face to face.

L.B., J.G. and I.M.M. share the Intellectual Property Right to BTSteps, and I.M.M. shares the IPR to FearFighter.
C 2007 S. Karger AG, Basel

0033-3190/07/0764-0193\$23.50/0

Fax +4161306 1234

E-Mail karger@karger.ch

www.karger.com
Accessible online at: www.karger.com/pps
Dropout rates soar, however, if Internet access to CCBT is open and unmonitored [1].

The coming of age of CCBT as a way of delivering mental health care was recently recognized by its first approval by a government agency. That approval came from the National Institute for Clinical Excellence (NICE), the English regulatory body. NICE [3] now recommends 2 CCBT systems for the National Health Service - Beating the Blues for depression and FearFighter for panic and phobic disorders.

Few CCBT systems have dealt with obsessive-compulsive disorder (OCD). There have been case reports of CCBT with OCD $[4,5]$ and a pilot study of vicarious exposure by CCBT $[6,7]$. Most CCBT research on OCD so far has concerned the BTSteps (Behavior Therapy Steps) system [8], which Tumur et al. [9] and McCrone et al. [10] refer to in this issue.

The BTSteps system delivers its CCBT by telephone via a computer-driven interactive voice response (IVR) system which helps the user plan and carry out the selfdirected exposure and ritual prevention that are core features of effective CBT for OCD. The patient directs the computer IVR interview by key presses on the phone, usually at home and mostly outside usual working hours. (In Britain BTSteps has been adapted for use at home on the Internet and is available under the name OCFighter to avoid confusion with a well-known telephone company). 
The review by Tumur et al. [9] of CCBT for OCD arises from part of a report [11] which NICE commissioned from them. Largely based on that report, the NICE approved CCBT for panic/phobia and depression, but not for OCD. An Appeal challenged the judgment regarding OCD, after which NICE finally accepted the 'absolute clinical efficacy' of BTSteps for OCD [12]. Tumur et al. are to be congratulated on their present review's correction of errors which were present in their original report to NICE and were largely understandable given the novel nature of IVR self-help and its mode of clinical application.

A couple of misunderstandings remaining in their present review deserve comment. First, it says 'the use of supplementary CCBT is clearly worth exploring. In reality, in BTSteps studies CCBT was not supplementary but rather was the primary mode of delivering CBT for OCD. What was supplementary was the brief human support on a helpline that BTSteps users received in some studies. The bulk of the patient's interaction was with the BTSteps computer - the patient had a total of only an hour or so of helpline support over 3 months of BTSteps use in some studies, and none in others. In an RCT, BTSteps users did best if they were given support in 5- to 10-min scheduled phone calls totalling a mean of 76 min over 3 months rather than left to seek support only if desired.

In the multicentre RCT of BTSteps summarized by Tumur et al. [9], users had no human support at all, though ethical committee approval required that all patients including controls should see a clinician several times for a few minutes for safety reasons - the clinician gave no CBT. In that RCT BTSteps users cut daily total time spent in rituals and obsessions by $3.4 \mathrm{~h}-5$ times more than controls did, and about as much as did patients having face-to-face CBT. BTSteps also cost $264 \%$ less than face-to-face CBT for each unit of improvement on the Yale-Brown Obsessive-Compulsive Scale [10]. As Tumur et al. [9] note, BTSteps had a consistently better outcome on almost every measure than did a control relaxation group. Compared to face-to-face CBT, BTSteps was less effective on some measures, but as effective as faceto-face CBT on almost every measure where patients had begun some exposure homework (64\% of patients). Faceto-face therapy may thus act mainly as a motivator to start self-exposure and self-imposed ritual prevention.

A second misapprehension is the claim of Tumur et al. [9] that 'it was not possible to quantify the exact number and length of the CCBT sessions as (the) IVR system was not designed for a fixed number of sessions'. In fact one of the many strengths of BTSteps is that its central IVR computer tracks each call (session) of the patient to BTSteps and the timing, duration and content of that call, the total number and mean duration of all calls, and the patient's IVR self-ratings of outcome at intervals. The BTSteps computer tracks each user by their anonymous code number and stores no personal data. Only a health authority licensed to use BTSteps can link patients' anonymous code numbers to personal data. CCBT by BTSteps can thus give a more detailed, efficient, automatic and anonymized audit of what happens during therapy than is possible in face-to-face care. The same applies to Internet systems like FearFighter.

There is always a need for improvement in any mode of delivering care, but already computer-aided self-help can 'widen the access to CBT in general and considerably shorten clinician-guided care' [9]. CCBT designed for patients can also expedite the education of professionals about CBT, as was found in 2 RCTs in nurses and medical students [1]. Major issues now are how health care systems round the world can best fund and organize computer-aided self-help.

Time and education is needed for therapists, patients, health authorities, health insurers and government departments of health to adapt to the new way of delivering care by CCBT. It augurs well for delivering consistently high-standard care which many patients prefer. A large number want to access CBT at home by CCBT rather than in a clinic, for reasons of greater confidentiality, lessening of stigma and reduction of time needed to travel to a therapist, among others. For the first time, affordable access to effective self-help may be in sight for all OCD, panic/phobia and depression sufferers who want it. Giving wide and early access to it could reduce chronic burden on patients, families and society. Independent research is now needed into how public health benefits can best be reaped from computer-aided psychotherapy. 


\section{References}

1 Marks IM, Cavanagh K, Gega L: HandsOn Help. Computer-Aided Psychotherapy. Maudsley Monograph No 49. Hove, Psychology Press, 2007.

2 Emmelkamp PMG: Technological innovations in clinical assessment and psychotherapy. Psychother Psychosom 2006;74:336343.

3 National Institute for Clinical Excellence (NICE): Technology Appraisal TA097. 2006. http://www.nice.org.uk/page. $a s p x ? 0=t a 097$ (accessed February, 2006).

4 Baer L, Minichiello WE, Jenike MA: Use of a portable-computer program in behavioral treatment of OCD. Am J Psychiatry 1987; 144:1101.
5 Baer L, Minichiello WE, Jenike MA, Holland A: Use of a portable-computer program to assist behavioral treatment in a case of OCD. J Behav Ther Exp Psychiatry 1988;19:237240.

6 Clark A, Kirkby KC, Daniels BA, Marks IM: A pilot study of computer-aided vicarious exposure for OCD. Aust NZ J Psychiatry 1998;32:268-275.

7 Kirkby KC, Berrios GE, Daniels BA, Menzies RG, Clark A, Romano AJ: Process-outcome analysis in computer-aided treatment of OCD. Compr Psychiatry 2000;41:259-265.

8 Greist JH, Marks IM, Baer L, Kobak KA, Wenzel KW, Hirsch MJ, Mantle JM, Clary CM: Behaviour therapy for obsessive compulsive disorder guided by a computer or by a clinician compared with relaxation as a control. J Clin Psychiatry 2002;63:138-145.
9 Tumur I, Kaltenthaler E, Ferriter M, Beverley C, Parry G: Computerised cognitive behaviour therapy for obsessive compulsive disorder: a systematic review. Psychother Psychosom 2007;76:196-202 .

10 McCrone P, Marks IM, Greist JH, Baer L, Kobak KA, Wenzel KW, Hirsch MJ: Cost-effectiveness of computer-aided behaviour therapy for OCD (letter). Psychother Psychosom 2007;76:248-249.

11 National Institute for Clinical Excellence (NICE), 2005. http://www.nice.org.uk/page. aspx?o $=247657$.

12 National Institute for Clinical Excellence (NICE): NICE's Appeal outcome letter, 15 February 2006. 